\title{
Craniocervical Artery Dissection: Clinical and Imaging Mid-term Follow Up
}

Seon-Kyu Lee ${ }^{1^{*}}$, Kiron Thomas ${ }^{2}$, Nicholas Tsapatsaris ${ }^{3}$, Fawad Al-Mufti ${ }^{6}$, Thomas Piemonte ${ }^{4}$ Edward Jewel $^{5}$ and Kinan Hreib $^{2}$

${ }^{1}$ Department of Radiology, University of Chicago, USA

${ }^{2}$ Department of Neurology, Lahey Clinic Medical Center, USA

${ }^{3}$ Department of Vascular Medicine, Lahey Clinic Medical Center, USA

${ }^{4}$ Department of Cardiology, Lahey Clinic Medical Center, USA

${ }^{5}$ Department of Vascular Surgery, Lahey Clinic Medical Center, USA

${ }^{6}$ Department of Neurology, SUNY Stony Brook, USA

Corresponding author: Seon-Kyu Lee, Director, University of Chicago, 5841 St. Maryland Avenue, MC2026, Chicago, IL 60637, USA, Tel: (773) 702-3654; Fax: (773) 702-1161; E-mail: sklee@uchicago.edu

Rec date: Oct 07, 2016, Acc date: Oct 18, 2016, Pub date: Oct 22, 2016

Copyright: () 2016 Lee SK, et al. This is an open-access article distributed under the terms of the Creative Commons Attribution License, which permits unrestricted use, distribution, and reproduction in any medium, provided the original author and source are credited.

\begin{abstract}
Background and objective: To analyze the temporal evolution of imaging findings and clinical follow up in craniocervical arterial dissection (CAD) patients.

Methods: 32 patients ( $\mathrm{M}: \mathrm{F}=23: 9$, mean age=49) with radiographic evidence of at least one of the suggestive imaging findings of CAD (arterial stenosis, arterial occlusion, luminal ectasia, pseudoaneurysm, and dissection flap) were performed. Median imaging follow up period was 89 days $(\mathrm{SD}=36.69)$. Clinical symptoms on presentation and follow up were also analyzed.
\end{abstract}

Results: 21 patients had spontaneous dissection (65.6\%). 11 had a history of trauma (34.4\%). 14 (43.8\%) patients presented with a stroke or TIA. Other presenting symptoms include headache $(n=4,12.5 \%)$, neck pain $(n=9$, $28.1 \%)$ and Horner's syndrome $(n=5,15.6 \%)$.

Involved arteries included Internal carotid artery $(n=21,65.6 \%)$, vertebral artery $(n=10,31.3 \%)$, and common carotid artery $(n=3,9.4 \%)$. Initial imaging findings included, arterial stenosis $(n=23,72 \%)$, arterial occlusion ( $n=6$, $19 \%)$, ectasis of the lumen $(n=7,22 \%)$, pseudoaneurysm $(n=12,38 \%)$, and dissection flap $(n=12,38 \%)$.

21 patients were managed with anti-coagulation and 3 with an antiplatelet agent. On follow up imaging, arterial stenosis was improved in $73.6 \%$ (14/19), worse in $5.3 \%$ and no interval change in $21.1 \%$. Regarding the pseudoaneurysm on presentation, no change in shape and size in $64 \%(7 / 11)$ and 2 patients developed new pseudoaneurysm. One patient developed a TIA $(3.7 \%, n=1 / 27)$ during follow up.

Conclusion: Post-dissection vascular imaging findings are dynamic. With medical management, more than $60 \%$ of the arterial stenosis lesions have improved within 3 months, and the risk of repeated neurological events were very low.

Keywords: Craniocervical dissection; Clinical follow up; Imaging follow up; CT angiography; MR angiography

\section{Introduction}

Craniocervical arterial dissection is an important cause of ischemic stroke in relatively young age group [1,2]. Pathological definition of craniocervical arterial dissection is formation of intramural hematoma in the arterial wall. The development of intramural hematoma can be resulted from either intimal tear or primary intramural hemorrhage $[3,4]$. The natural history of spontaneous craniocervical artery dissection is not entirely understood, but the outcome is considered generally good to excellent with less than $10 \%$ of overall mortality rate [5] On the other hand, about $5 \%$ of patients were left with major residual neurological sequelae, and $15 \%$ with minor residual deficits. [6-8].

Acute dissections are frequently treated with either antiplatelet agents or anticoagulation for a minimum of three months but duration of medical treatment has not been standardized and could be influenced by initial and follow up imaging studies.

Several imaging modalities, including Doppler ultrasonography, MR and CT angiography, are available to confirm the initial diagnosis of cervical arterial dissection, and to determine treatment planning and options [9]. However, there are not many reports available regarding progression of initial imaging findings and associated clinical symptoms. 
Page 2 of 6

The purpose of this study is to analyze the temporal evolution of imaging findings and related clinical follow up in craniocervical arterial dissection patients.

\section{Patients and Methods}

This retrospective imaging review study was approved by the institutional review board.

From 2002 to 2009, 32 patients ( $\mathrm{M}$ : $\mathrm{F}=23$ : 9, mean age $=49$, $\mathrm{SD}=16.47$ ) with at least one of the following imaging findings of carotid dissection on either CT angiography or MR angiography were included. Those imaging findings were: 1) arterial stenosis 2) arterial occlusion 3) ectasis of the lumen 4) pseudoaneurysm and 5) dissection flap.

The temporal evolution of above 5 imaging findings was determined by consensus of 2 neuroradiologists. Patients who do not have any available follow up CT angiography nor $\mathrm{MR}$ angiography were excluded.

\section{Definition of imaging findings}

Arterial stenosis was defined as more than $20 \%$ of arterial luminal narrowing comparing to normal appearing proximal segment of parent artery. However, we did not measure individual arterial stenosis degree. Segmental dilatation (more than $5 \mathrm{~mm}$ length) of a vessel with maximum diameter of the lumen showed more than $20 \%$ than normal appearing proximal segment of parent artery was defined as ectasis as opposed to focal (less than $5 \mathrm{~mm}$ length) widening or ballooning which was considered pseudoaneurysm.

\section{Clinical and imaging follow up}

Clinical and imaging follow ups were available in 27 patients (27/32, $84 \%)$. There were 4 patients who had 1 follow up imaging study, 15 patients who had 2 follow ups and remained 8 patients had more than 3 follow up imaging studies.

The last clinical follow up was performed from 3 days to 1916 days after the initial diagnosis [mean=557.1 days (about 1.5 years), median=350days (about 11 months)].

\section{CT Angiography technique}

$\mathrm{CT}$ angiography was performed from aortic arch to the vertex with multi-detector CT scanner (either 8 slices or 64 slices) with following parameters: FOV $19-20 \times 23-24 \mathrm{~cm}$, Matrix size $512 \times 512$, Slice thickness $=1.25 \mathrm{~mm} \sim 0.63 \mathrm{~mm}$. Multiplanar reconstruction including axial, sagittal and coronal plane as well as $3 \mathrm{D}$ reconstructions using volume rendering technique was also performed.

\section{MR Angiography technique}

1.5 T MRI scanner with standard head and neck surface coil was used. The MR angiography protocol included axial fat suppression T1 weighted (T1-FS) images from carotid bifurcation to the skull base and diffusion weighted images of brain.

The T1-FS images were obtained with FOV $18 \mathrm{~cm}$, Matrix $256 \times 192$, slice thickness $3 \mathrm{~mm}$, gap $3.5 \mathrm{~mm}$ and MR angiography was performed with FOV $28 \mathrm{~cm}$, Matrix $320 \times 190$, Slice thickness $1.4 \mathrm{~mm}$, Space 0.7 $\mathrm{mm}$. For the Gadolinium enhanced head and neck MR angiography, about $14 \sim 20 \mathrm{cc}$ of Gadolinium was infused intravenously.

\section{Statistical analysis}

The statistical significances of imaging finding changes on follow up scan were assessed using 95\% Confidence Interval (CI).

\section{Results}

\section{Clinical presentations}

21 patients were diagnosed as spontaneous dissection (12/32, $65.6 \%)$, on the other hand, 11 had a history of either significant or suggestive trauma for possible cervico-cephalic vascular injury (11/32, $34.4 \%) .14$ of the 32 patients (43.8\%) presented with a stroke or TIA.

In 4 patients $(4 / 32,12.5 \%)$, headache was the only presenting complaint. $9(28.1 \%)$ patients had lancinating neck pain with or without headache. Horner's syndrome was noticed in only 5 patients $(5 / 32,15.6 \%)$.

\section{Clinical management}

Clinical follow up with medical management regimen were available in 27 patients. Among them, 21 patients were treated with anticoagulation alone, 4 with antiplatelet agent alone and 2 patients were treated with both anticoagulation and antiplatelet agent.

One patient who received anticoagulation alone underwent subsequent carotid endarterectomy due to expanding pseudoaneurysm. Another patient who was treated antiplatelet agent alone underwent angioplasty and stenting due to progressive arterial stenosis. During the follow up period, only one patient among 27 patients developed a TIA $(3.7 \%, n=1 / 27)$.

\section{Anatomical locations}

There were 19 internal carotid artery (ICA) dissections (19/32, 59.4\%), 9 vertebral artery (VA) dissections (9/32, 28.1\%), 2 common carotid artery (CCA) dissections $(2 / 32,6.3 \%)$ and 2 bilateral ICA dissections $(2 / 32,6.3 \%)$. All common carotid artery dissections and bilateral ICA dissections had history of trauma.

Dissections involving right ICA, left ICA, right VA, left VA, right CCA and left CCA were $12,7,3,6,0$, and 2 respectively. $38.1 \%(8 / 21)$ patients with ICA dissections and $60 \%(6 / 10)$ with vertebral artery dissections presented with a stroke or TIA.

\section{Initial imaging findings}

On presentation, among 32 patients, arterial stenosis was seen in 23 (72\%), arterial occlusion in $6(19 \%)$, ectasis of the lumen was noted in $7(22 \%)$, pseudoaneurysm in $12(38 \%)$, and dissection flap in $12(38 \%)$.

\section{Follow up of Imaging findings}

In 27 patients (84\%), at least one follow up vascular imaging study was available (mean $=1.5$ years). Among 23 patients who had "stenosis" on initial imaging study, 19 patients had at least one follow up imaging studies. On follow up, arterial stenosis improved in 73.6\% (14/19) [95\% CI: $48.5 \%$ to $89.9 \%$ ], worse in $5.3 \%$ (1/19) [ $95 \%$ CI: $0 \%$ to $28.1 \%$ ] and showed no interval change in $21.1 \%$ (4/19) [95\% CI: $6.9 \%$ to $46.1 \%$ ].

The mean imaging follow up period which demonstrated luminal narrowing improvement was 79 days (median $=89$ days, $S D=36.69$ ) (Figure 1 and Table 1). 
Citation: Lee SK, Thomas K, Tsapatsaris N, Al-Mufti F, Piemonte T, et al. (2016) Craniocervical Artery Dissection: Clinical and Imaging Mid-term Follow Up . J Neurol Disord 4: 308. doi:10.4172/2329-6895.1000308

Page 3 of 6

Regarding patients who had pseudoaneurysm on initial imaging study, there were 11 patients who had at least one follow up imaging study. The pseudoaneurysm showed no change in shape and size in $63.6 \%(7 / 11)$ [95\% CI: $31.6 \%$ to $87.6 \%$ ], larger in size in $27.3 \%(3 / 11)$ [95\% CI: $7.3 \%$ to $60.7 \%$ ], smaller in size in $9 \%$ (1/11) [95\% CI: $0.5 \%$ to 42.9\%] (Figure 2).
Two among 13 patients who did not have pseudoaneurysm on initial imaging study developed a new pseudoaneurysm at the dissection site on follow up imaging $(2 / 13,15.4 \%)$ (Figure 2 ).
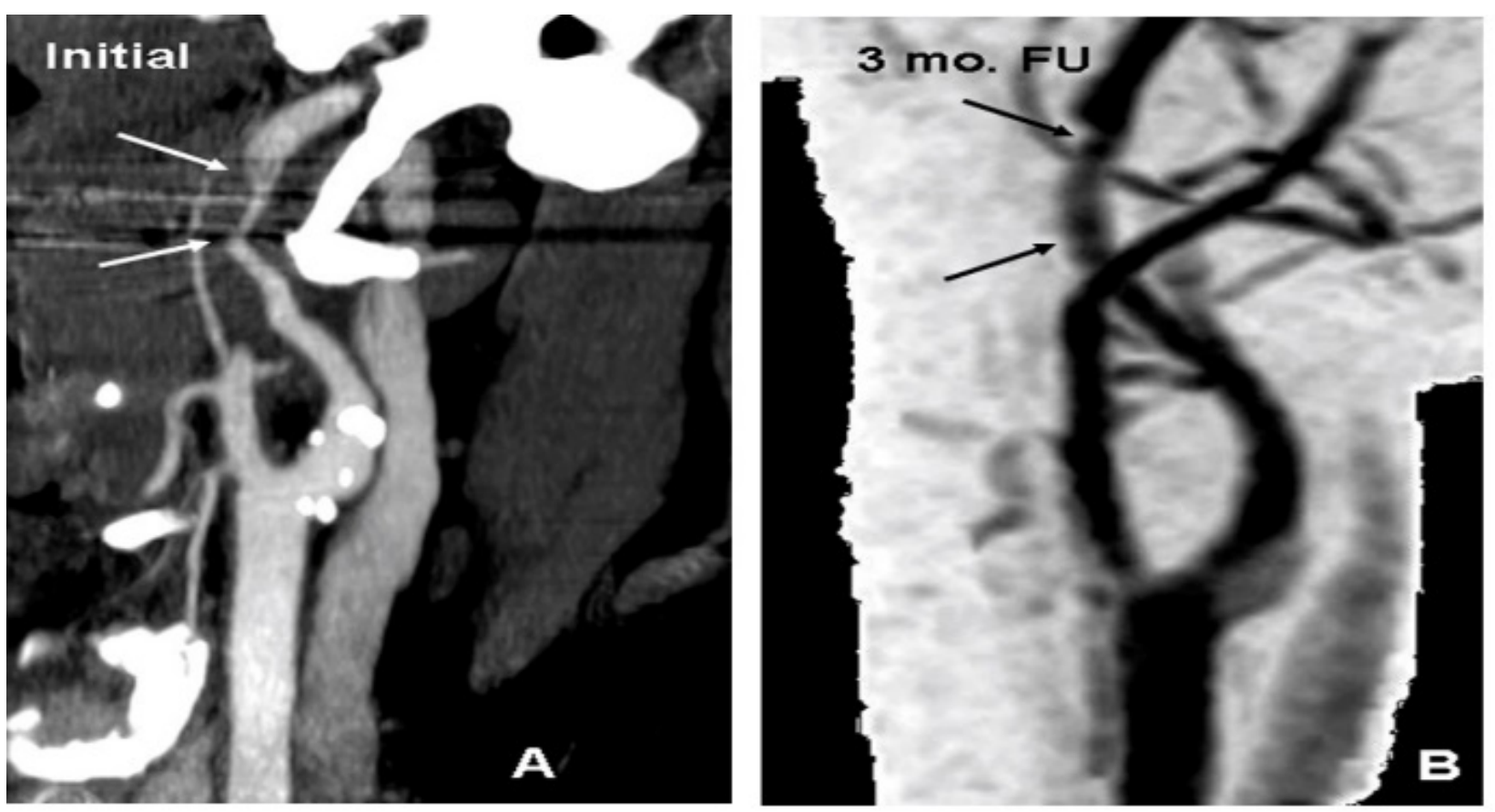

Figure 1: Improvement of Stenosis on Follow Up Imaging (42 y-o male with sudden development of left side visual loss.) Initial CTA (A) showed about $50 \%$ to $60 \%$ left ICA stenosis (arrows). 3 month follow up Gd enhanced MRA (B) showed near total recanalization of previously stenotic segment.
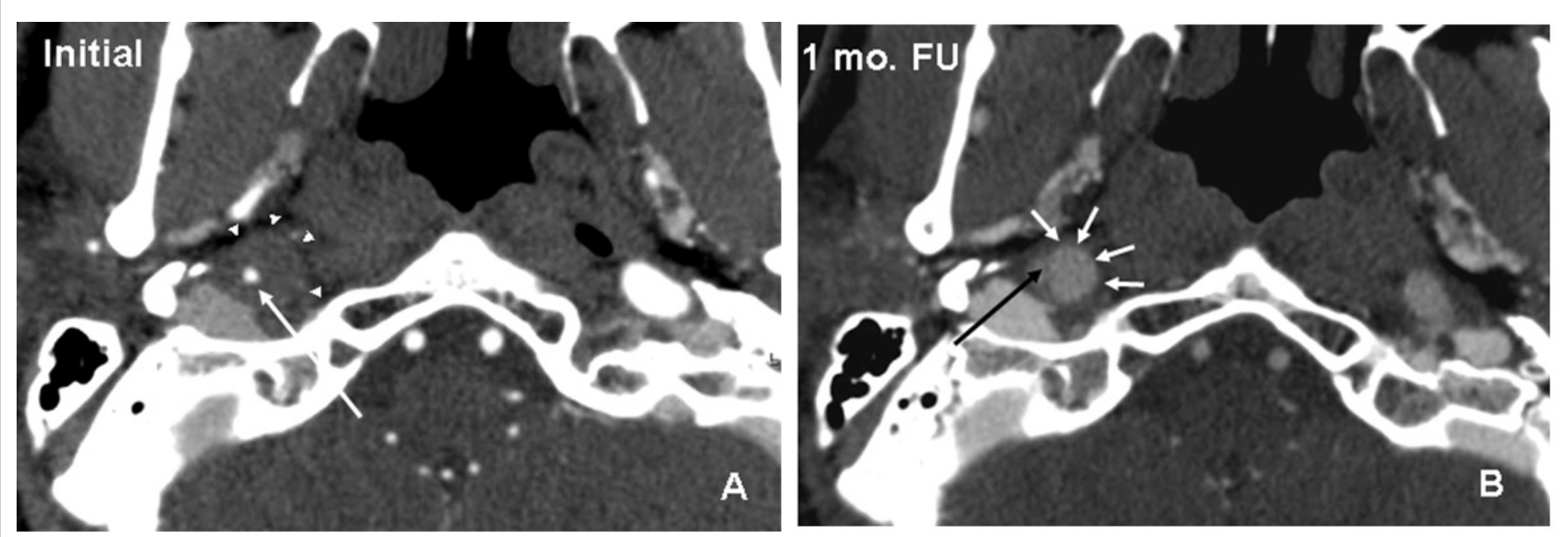

Figure 2: Development of pseudoaneurysm from intramural hematoma (57 y-o male presented with right side Horner's syndrome and right neck pain). Initial CTA (A) shows significant wall thickening of distal right ICA (white arrow heads) which represents intramural hematoma with luminal stenosis (white arrow). On 1 month follow up CTA (B), the wall thickening has been resolved and development of pseudoaneurysm (white arrows) with stenotic parent artery (black arrow). 
Citation: Lee SK, Thomas K, Tsapatsaris N, Al-Mufti F, Piemonte T, et al. (2016) Craniocervical Artery Dissection: Clinical and Imaging Mid-term Follow Up . J Neurol Disord 4: 308. doi:10.4172/2329-6895.1000308

Page 4 of 6

There were 6 patients who had complete occlusion of affected artery on presentation and in 4 patients; at least one follow up imaging study was available. Among them, $75 \%$ (3/4) [95\% CI: $21.9 \%$ to $98.7 \%$ ] demonstrated at least partial recanalization of previously occluded parent artery. Specifically, there was 1 complete recanalization $(1 / 4$, $25 \%)$ (Figure 3 ) and 2 partial recanalization $(2 / 4,50 \%)$ but 1 remained occluded on follow up scan.
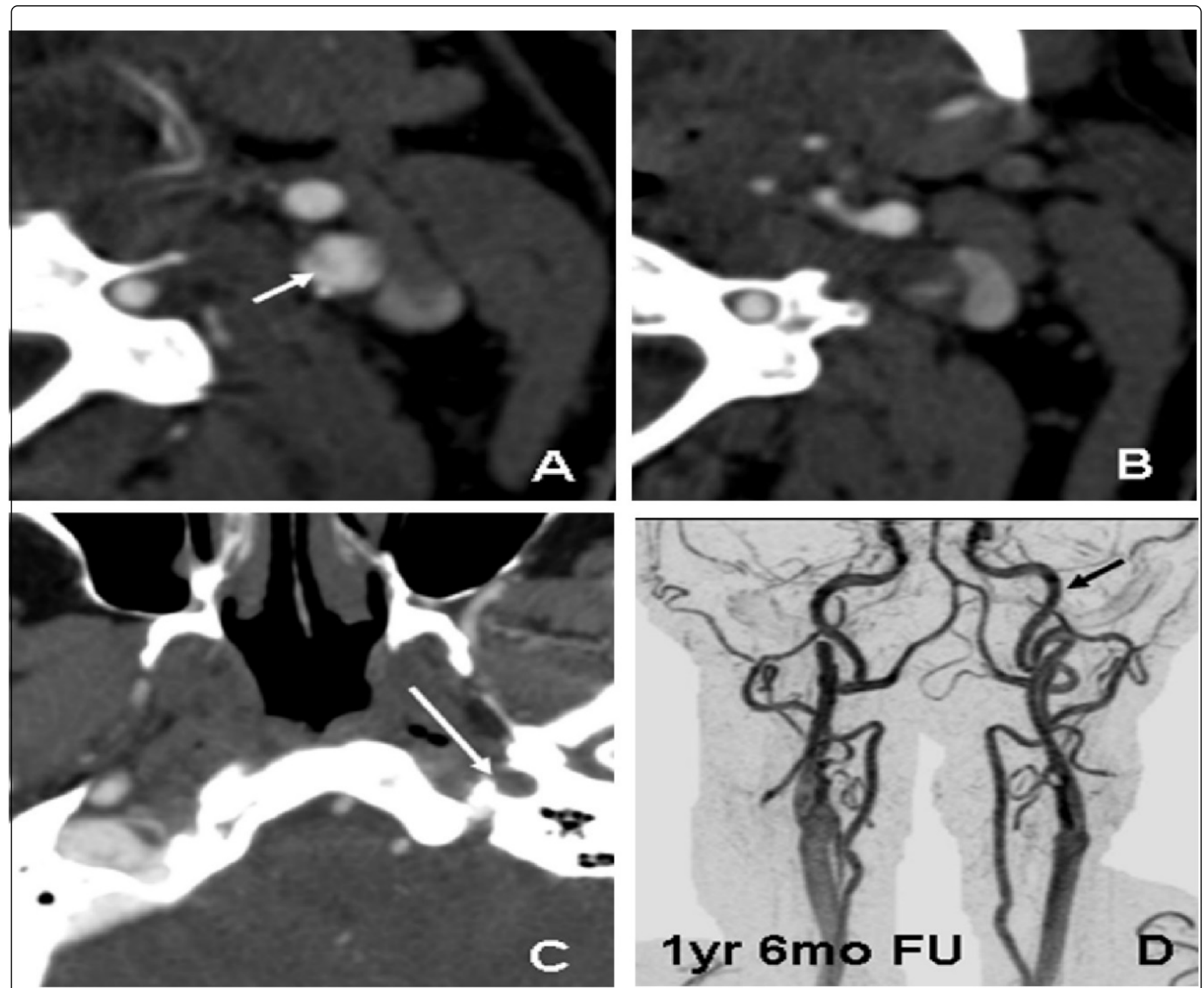

Figure 3: Complete recanalization of occluded segment (49 y-o male presented with sudden onset of right arm and leg weakness). Initial CTA (A to C) showed complete occlusion of the left ICA. There is subtle visualization of intimal flap (white arrow on A) and non-opacification of left ICA (white arrow on C). One and half year follow up Gadolinium enhanced MRA (D) showed complete recanalization of the left ICA (black arrow).

\section{Discussion}

In our series, about $60 \%$ of arterial stenosis improved $(14 / 23$, $60.9 \%)$, and $75 \%$ of completely occluded segment also have recanalized at least partially $(3 / 4,75 \%)$. About $60 \%$ of arterial pseudoaneurysm did not show significant interval change $(7 / 12,58 \%)$ on follow up imaging and $15.4 \%$ of patient developed a new pseudoaneurysm on the dissected segment.
The improvement of stenosis has been reported in $40 \%$ to $80 \%$ of patients $[7,10,11]$. In one study, a completely occluded or near occluded artery on presentation is reported as less likely to recanalize $[12,13]$, however, other studies showed that the recanalization rates of even completely occluded arteries as $42 \%$ to $69 \%$ [14-16]. In our series, $75 \%$ of completely occluded arteries showed either complete or partial recanalization on follow up. Thus, our results support that the initial presentation of arterial stenosis will likely improve with appropriate medical management. Moreover, significant number of even 
completely occluded arteries may also reanalyze on follow up imaging study.

\begin{tabular}{|c|c|c|c|c|c|c|c|c|}
\hline \multirow[t]{2}{*}{ Findings } & \multicolumn{2}{|c|}{ Initial Imaging } & \multicolumn{2}{|c|}{$\begin{array}{l}\text { TIA or Stroke } \\
\text { Presentation }\end{array}$} & \multirow[t]{2}{*}{$\begin{array}{l}\text { Imaging FU } \\
\text { (Pt.Number) }\end{array}$} & \multicolumn{3}{|l|}{ FU imaging } \\
\hline & $N($ total=32) & $\%$ & $\mathbf{N}$ & $\%$ & & Intv Changes & $\mathbf{N}$ & $\%$ \\
\hline Stenosis & 23 & $72 \%$ & 11 & $48 \%$ & 19 & $\begin{array}{l}\text { Imprv } \\
\text { Unchg } \\
\text { Worse }\end{array}$ & $\begin{array}{l}14 \\
4 \\
1\end{array}$ & $\begin{array}{l}73.6 \% \\
21.1 \% \\
5.3 \%\end{array}$ \\
\hline Occlusion & 6 & $19 \%$ & 3 & $50 \%$ & 4 & $\begin{array}{l}\text { CmplRecan } \\
\text { PartRecan } \\
\text { Unchg }\end{array}$ & $\begin{array}{l}1 \\
2 \\
1\end{array}$ & $\begin{array}{l}25 \% \\
55 \% \\
25 \%\end{array}$ \\
\hline Ectasia & 7 & $21.9 \%$ & 3 & $33 \%$ & 7 & $\begin{array}{l}\text { Imprv } \\
\text { Unchg } \\
\text { Worse }\end{array}$ & $\begin{array}{l}1 \\
5 \\
1\end{array}$ & $\begin{array}{l}14.3 \% \\
71.4 \% \\
14.3 \%\end{array}$ \\
\hline Pseudoaneurysm & 12 & $38 \%$ & 3 & $25 \%$ & 11 & $\begin{array}{l}\text { Larger } \\
\text { Unchg } \\
\text { Smaller } \\
\text { New }\end{array}$ & $\begin{array}{l}3 \\
7 \\
1 \\
2 / 13\end{array}$ & $\begin{array}{l}27.3 \% \\
63.6 \% \\
9 \% \\
15.4 \%\end{array}$ \\
\hline Intimal Flap & 12 & $38 \%$ & 6 & $38 \%$ & 7 & Unchg & 7 & $100 \%$ \\
\hline
\end{tabular}

TIA: Transient Ischemic Attack, Pt.:Patient, FU:Follow Up, Intv Changes: Interval Changes, Imprv:Improvement, Unchg:Unchanged, CompIRecan:Complete Recanalization, PartRecan:Partial Recanalization

Table 1: Evolution of Imaging findings after craniocervical arterial dissection.

Pseudoaneurysm and intimal flap tend to remain stable once developed. However, new pseudoaneurysm can develop as the wall hematoma resolves. In addition, established pseudoaneurysm can increased in size as wall hematoma resolves on follow up.

Thus follow up imaging study in craniocervical arterial dissection is very important to monitor temporal evolution of dissected arterial segment even completely occluded one.

The imaging appearance of thickening of the dissected arterial wall which represents intramural hematoma and luminal stenosis are representing arterial wall hematoma [3]. The temporal improvement of arterial stenosis including complete occlusion might also be related to the degree and speed of wall hematoma resorption process. Therefore, if we can manage the resorption of wall hematoma relatively faster and more complete, the luminal gain would be faster and more complete. However, as far as authors know, there is no report showing any significant differences of arterial wall hematoma resorption speed and effectiveness between anti-platelet and anti-coagulation therapy. Our study could not draw any conclusion either regarding this issue since most of our cases were managed with anti-coagulation. Based on previous studies $[7,8,10,12]$, the speed and completeness of arterial wall hematoma resorption does not appear to be affected by the difference of anti-thrombotic therapy.

Stent assisted angioplasty has been regarded as an option for craniocervical arterial dissection management especially when a patient presented with significant carotid artery stenosis assuming relatively higher risk of ipsilateral ischemic stroke. However, regardless of the degree of carotid artery stenosis and clinical symptoms on presentation, both prior studies $[6,8]$ and our results $(1 / 26,3.8 \%)$ support that the risk of developing recurrent neurological symptoms are very low in patients who have been medically managed. Therefore, revascularization procedures such as stent assisted angioplasty should be considered only when a patient shows recurrent neurologic symptoms in spite of anti-thrombotic therapy. Since neither anticoagulation nor antiplatelet therapy seem to alter the natural course of the wall hematoma resorption process, when a patient is symptomatic despite medical treatment, active revascularization procedure may need to be considered promptly. However, if a patient is asymptomatic but shows significant luminal stenosis on imaging study (for example, more than $70 \%$ stenosis), we recommend continuing anti-thrombotic therapy since the recurrence rate of neurological symptom appears to be very low and most of luminal stenosis will get better on follow up.

There are limitations on our study. Since our study is a retrospective in nature, it may not reflect the actual progression of imaging findings after craniocervical dissections. However, our results were very consistent to other reports regarding the imaging progression after the diagnosis of craniocervical dissection. Second, our follow up imaging was performed empirically as a part of our clinical protocol which was about 3 months after the initiation of medical management, so the luminal improvement was only identifiable around that period of time ( $m e a n=79$ days, median $=89$ days). Thus, the luminal improvement after initiation of medical management could have happened earlier time period than our results. In addition, our imaging follow up period showed wide standard deviation thus estimation of the time period for interval imaging finding change is challenging. 
Citation: Lee SK, Thomas K, Tsapatsaris N, Al-Mufti F, Piemonte T, et al. (2016) Craniocervical Artery Dissection: Clinical and Imaging Mid-term Follow Up . J Neurol Disord 4: 308. doi:10.4172/2329-6895.1000308

Page 6 of 6

Unfortunately, the best management strategy for craniocervical arterial dissection remains controversial and is based on applying rational physiological principles. There is not a single randomized trial or even a large registry to inform management. Our study along with other observational studies can be used as a basis for designing such trials. Given the increasing recognition of craniocervical arterial dissection, such trials are long overdue.

In conclusion, post-dissection vascular imaging findings are dynamic. With medical management, more than half of the arterial stenosis after craniocervical dissection improved within a relatively short period of time (about 2.5 months) and the risk of repeated neurological event appears to be very low.

\section{References}

1. Bougousslavsky J, Regli F (1987) Ischemic stroke in adults younger than 30 years of age. Arch Neurol 44: 479-482.

2. Leys D, Bandu L, Henon H, Lucas C, Mounier-Vehier F, et al. (2002) Clinical outcomes in 287 consecutive young adults (15 to 45 years) with ischemic stroke. Neurology 59: 26-33.

3. Schievink WI (2001) Spontaneous dissection of the carotid and vertebral arteries. N Engl J Med 344: 898-906.

4. Friedman WA, Day AL, Quisling RG, Sypert GW, Rhoton AL (1980) Cervical carotid dissecting aneurysms. Neurosurgery 7: 207-214.

5. Fischer U, Lederman I, Nedeltchey K Meier N, Gralla J, et al. (2009) Quality of life and survivors after cervical artery dissection. J Neurol 256 443-449.

6. Beletsky V, Nadareishvili Z, Lynch J, Shuaib A, Woolfenden A, et al. (2003) Canadian Stroke Consortium. Cervical arterial dissection; time for a therapeutic trial? Stroke 34: 2856-2860.
7. Arauz A, Hoyos L, Espinoza C, Cantú C, Barinagarrementeria F, et al. (2006) Dissection of cervical arteries: long term follow up study of 130 consecutive cases. Cerebrovasc Dis 22: 150-154.

8. Georgiadis D, Arnold M, Von Buedingen HC (2009) Aspirain vs anticoagulation in carotid artery dissection. A study of 298 patients. Neurology 72: 1810-1815.

9. Provenzale JM, Sarikaya B (2009) Comparison of test performance characteristics of MRI, MR angiography, and CT angiography in the diagnosis of carotid and vertebral artery dissection: a review of the medical literature. AJR Am J Roentgenol 193: 1167.

10. Leclerc X, Lucas C, Godefroy O (1998) Helical CT for the follow up of cervical internal carotid artery dissections. Am J Neuroradiol 19: 831-837.

11. Pozzati E, Giuliani G, Acciarri N, Nuzzo G (1990) Long term follow up of occlusive cervical carotid dissection. Stroke 21: 528-531.

12. Schwartz N, Vertinsky A, Hirsch K, Albers G (2009) Clinical and radiographic natural history of cervical artery dissections. J Stroke Cerebrovasc Dis 18: 416-423.

13. Weimar C, Kraywinkel K, Hagemeister C (2010) Recurrent stroke after cervical artery dissection. J Neurol Neurosurg Psychiatry 8: 869-873.

14. Sengelhoff C, Nebelsieck J, Nassenstein I (2008) Neurosonographical follow up in patients with spontaneous cervical artery dissection. Neurol Res 30: 687-689.

15. Steinke W, Rautenberg W, Schwartz A, Hennerici M (1994) Noninvasive monitoring of internal carotid artery dissection. Stroke 25: 998-1005.

16. Caso V, Paciaroni M, Corea F (2004) Recanalization of cervical artery dissection: Influencing factor and role in neurological outcome. Cerebrovasc Dis 17: 93-97. 